\title{
Multiscale Hybridization of Natural Silk-Nanocellulose Fibrous Composites With Exceptional Mechanical Properties
}

\author{
Jungju Eom ${ }^{1}$, Subong Park ${ }^{2}$, Hyoung-Joon Jin ${ }^{1 *}$ and Hyo Won Kwak ${ }^{3,4 *}$ \\ 1 Department of Polymer Science and Engineering, Inha University, Incheon, South Korea, ${ }^{2}$ Fisheries Engineering Division, \\ National Institute of Fisheries Science, Busan, South Korea, ${ }^{3}$ Department of Forest Sciences, Seoul National University, \\ Seoul, South Korea, ${ }^{4}$ Research Institute of Agriculture and Life Sciences, College of Agriculture and Life Sciences, Seoul \\ National University, Seoul, South Korea
}

\section{OPEN ACCESS}

Edited by: Antonella Motta, University of Trento, Italy

Reviewed by:

Marina Scarpa

University of Trento, Italy

Veronique Michaud,

Federal Institute of Technology

in Lausanne, Switzerland

*Correspondence: Hyoung-Joon Jin

hijin@inha.ac.kr

Hyo Won Kwak

bk0502@snu.ac.kr

Specialty section:

This article was submitted to

Polymeric and Composite Materials,

a section of the journal

Frontiers in Materials

Received: 20 December 2019

Accepted: 30 March 2020

Published: 15 May 2020

Citation:

Eom J, Park S, Jin H-J and Kwak HW (2020) Multiscale

Hybridization of Natural

Silk-Nanocellulose Fibrous Composites With Exceptional

Mechanical Properties.

Front. Mater. 7:98

doi: 10.3389/fmats.2020.00098
Because of the crisis of the petrochemical era, environmentally friendly natural polymers and related material processes are receiving great attention. Cellulose and silk are typical fibrous materials that consist of polysaccharides and proteins, respectively, and have excellent mechanical properties and high physicochemical stabilities due to their unique self-assembly-based hierarchical structures. In this study, highly stable high-strength silk fiber (SF)-cellulose nanofibril (CNF) composites were prepared by the hierarchical fusion of micrometer-scale SFs and nanometer-scale CNFs. This manufacturing process is cost-effective because the raw materials (SFs and CNFs) are used as is. It is an eco-friendly process because it does not require the use of organic solvents or toxic reagents. In addition, it is an energy-efficient process because heat fusion $\left(120^{\circ} \mathrm{C}\right)$ takes only 10 min. The results of the Direct Red 80 staining experiments confirm that up to 15 wt.\% CNFs were added to the SF nonwoven. With the increase in the CNF amount, the nanometer-scale CNFs form a coating on the micro-scale SFs. At the same time, the CNFs form bonds with the SFs and increase the interfibrillar bonding strength of the CNF-coated SFs. Therefore, the mechanical properties of the SF/CNF composite and its stability in the water environment rapidly increase with increasing CNF concentration. In the case of SF/CNF15, the mechanical and impact strengths increase by 110 and $228 \%$, respectively, compared with SF nonwoven without CNF. In addition, as CNFs are introduced, hydrophobicity of the surface and bulk of the SF/CNF composite can be imparted, thereby maintaining its structural stability in the water environment. This eco-friendly SF/CNF composite can be widely used as reinforcement preform of fiberreinforced plastics as well as for other applications in the fibrous composite industry.

Keywords: silk fibroin, cellulose nanofiber, fibrous composite, nonwoven, adhesion

\section{INTRODUCTION}

Silk is an attractive natural fibrous material with a unique hierarchical composite structure (Shao and Vollrath, 2002; Vollrath et al., 2013; Koh et al., 2015). Its molecular structure consists of repetitive amino acid sequences (Malay et al., 2016), which form a secondary $\beta$-sheet-driven protein structure by the surrounding environment to form silk nanofibrils. The self-assembled nanofibrils 
agglomerate to produce fibroin microfibers and, finally, a bicomponent nonwoven cocoon fabric in which sericin surrounds two fibroin strands (Keten et al., 2010; Omenetto and Kaplan, 2010; Asakura et al., 2015; Malay et al., 2016; Wang et al., 2018b). Based on its unique hierarchical structure, silk has excellent mechanical properties (Chen et al., 2012; Shah et al., 2014; Sanjay et al., 2018) and is resistant to physical attacks and harsh environmental conditions. In addition, silk cocoons produced by silkworms have the advantage that they occur under environmentally friendly process conditions such as room temperature and atmospheric pressure.

Because of their excellent biocompatibility and biodegradability as well as intrinsic mechanical rigidity, various regenerated silk materials have received increased attention (Han et al., 2017; Magaz et al., 2018; Yazawa et al., 2018; Zhang et al., 2018). However, regenerated silk materials have a significant disadvantage in that they are difficult to scale up (Lee et al., 2018). During the regeneration process, environmental problems occur because of the use of organic solvents. In addition, molecular weight decomposition occurs in natural silk, leading to the deterioration of its physical properties (Cho et al., 2012; Kim et al., 2014; Lee et al., 2018; Yin et al., 2019). To overcome the above-mentioned disadvantages and effectively utilize silk materials, untreated (no regeneration) natural silk fibers (SFs) should be used (Peng et al., 2019).

Fibrous and fiber preform composites, which are produced by using natural SF as raw material, consist of SFs and additional chemical binders that increase the bonding between the SFs (Song et al., 2009; Yuan et al., 2010; Shubhra et al., 2011; Guang et al., 2015). Their mechanical properties greatly depend on the interaction between the binder and fibers. To improve the interaction between the binder and fiber, chemical modifications (Dányádi et al., 2010; Guo et al., 2016; Lee et al., 2019) (i.e., ionic, electrostatic, polar, and van der Waals forces exerted on the fiber and adhesive) and physical modifications of the fiber surfaces (Song et al., 2010), which increase the roughness and specific surface area of the fibers, have been widely used (Adekunle, 2015). However, these modifications lead to the physicochemical damage of the fibers and thus the mechanical weakness of the product if the modification time is extensive (Baiardo et al., 2004; Carvalho et al., 2010; Favaro et al., 2010; Das et al., 2015; Pandey et al., 2015). Another approach to increasing the interaction between the fibers is to fabricate hairy and fuzzy fibers based on the adhesion of finely sized nanomaterials to the surface of the fibers (Juntaro et al., 2008; Pommet et al., 2008; Lee et al., 2012; Arévalo and Peijs, 2016; Fortea-Verdejo et al., 2016).

Multiscale hybridized materials have received great attention by the scientific and industrial communities during the last decade due to their synergistic effects originating from different morphological scales (Duan et al., 2017; Lin et al., 2017; Toprakcioglu et al., 2017; Hadadi et al., 2018). In the composite materials approach, multiscale hybridization is an important key to solve inherent weaknesses such as the deterioration of the mechanical properties due to the poor reinforcement and structural dismantling in wet or aqueous environments (Fortea-Verdejo et al., 2016). In the biomedical fields, the production of multiscale materials can be used to create an effective drug delivery system (Numata et al., 2012; Gao et al., 2019), enabling sustainable and ultrafast drug delivery. At the same time, based on the nano-topologies of the microsurfaces (Mozumder et al., 2011), superhydrophobic materials can be produced. In addition, injectable scaffolds with reinforced mechanical strengths can be prepared by incorporating nano and microparticles into three-dimensional hydrogels (Xiao et al., 2017). Therefore, the fabrication of multiscale hybridizing fibrous composites using natural microand nanoscale fibrous materials is a simple and environmentally friendly fabrication method with a synergistic structureproperty relationship.

Wood is the most abundant natural resource on Earth and a cellular hierarchical biocomposite (Eichhorn et al., 2010). The self-assembly of hydrogen bond-based repetitive glucose units results in the formation of elemental fibrils, which develop into microcrystalline cellulose (MCC) (Tayeb et al., 2018). The MCC forms a macroscopic biocomposite structure that reinforces the matrix consisting of lignin, hemicellulose, and wax, eventually forming a whole tree with a scale of tens of meters. Various natural raw materials can be obtained from such a complex hierarchical structure of woody biomass (Klemm et al., 2005, 2011; Cervin et al., 2013). Such raw materials have been used to manufacture paper, (Song et al., 2009) pulp, (Theng et al., 2015) and fiber, which greatly contributed to human development. Recently, the development of advanced materials using nanocellulose has been actively attempted because of the robust physicomechanical properties of nanocellulose, which are based on its unique morphology and crystalline structure (Theng et al., 2015; Amini et al., 2017; Saba et al., 2017b,a; Tayeb et al., 2018; Feng et al., 2019; Kwak et al., 2019b).

To fabricate such a natural multiscale hybridizing polymer fibrous composite, SFs produced by silkworms and cellulose nanofibrils (CNFs) obtained from the woody biomass were used as natural micro and nanoscale fibrous raw materials, respectively. In this study, natural polymeric hierarchical fibrous composites were fabricated by using filtration and thermal fusion methods typically used in conventional paper manufacturing. The successful fabrication of hierarchal nano and microscale hybridization structures was verified using dye stains and the interactions between the nano and microscale fibers were confirmed using disintegration observations. Finally, the mechanical properties and water stability depending on the $\mathrm{CNF}$ concentrations of the natural multiscale hybridized fibrous composites were determined.

\section{MATERIALS AND METHODS}

\section{Materials}

Silk cocoons of the Bombyx mori silkworm were kindly provided by the National Academy of Agricultural Science (NAAS, Korea). Bamboo fibers were obtained from Damyang (2-3 years old, Phyllostachys, Korea) and used as native cellulose fibers. Direct Red 80 was obtained from Tokyo Chemical Industry Co., Ltd., Japan. $\mathrm{Na}_{2} \mathrm{CO}_{3}$ was purchased from Sigma-Aldrich (Korea). 


\section{Degumming of Silk Fibers (SF)}

The silkworm cocoons were boiled for $30 \mathrm{~min}$ in an aqueous solution containing $0.02 \mathrm{M}$ sodium carbonate $\left(\mathrm{Na}_{2} \mathrm{CO}_{3},>99.0 \%\right.$, Sigma-Aldrich) to remove sericin and other impurities. After washing the samples several times with deionized water (DI) water, the resulting protein SFs were dried for 3 days at $25^{\circ} \mathrm{C}$. Next, the degummed SF fibers were wound onto a small mandrel to yield $6 \mathrm{~cm}$ long aligned fibers.

\section{Preparation of Cellulose Nanofibril (CNF)}

To remove the waxy material from the bamboo, $2 \mathrm{~g}$ of the bamboo fiber was dewaxed on a Soxhlet equipment (SESeries, Vinci Technologies, France) with a benzene/ethanol [2: $1(\mathrm{v} / \mathrm{v})$ mixture of] for $6 \mathrm{~h}$. The lignocellulosic material in the sample was then removed using a sodium chlorite $\left(\mathrm{NaClO}_{2}\right)$ solution at $75^{\circ} \mathrm{C}$ for $1 \mathrm{~h}$ and repeated same process five times until the color of the sample became white. The residual hemicellulose, starch and pectin were further removed at $90^{\circ} \mathrm{C}$ for $2 \mathrm{~h}$ with $2 \mathrm{wt} \%$ potassium hydroxide $(\mathrm{KOH})$. Thereafter, bamboo cellulose dispersion ( $0.05 \mathrm{wt} \%)$ was prepared by adjusting the concentration of the dispersion with distilled water. About $200 \mathrm{~mL}$ of the bamboo cellulose dispersion was placed in an ultrasonic generator (Kyungil Ultrasonic Co., Korea), and subsequent sonication ( $800 \mathrm{~W}, 4 \mathrm{~s}$ on/off pulse) was performed for $1 \mathrm{~h}$ to obtain disintegrated cellulose nanofibrils. In order to avoid temperature elevation during high intensity ultrasonic processing, sonication was performed in an ice/water cooling bath system. In order to confirm nanofibril preparation, morphological image of bamboo CNF was obtained using field emission scanning electron microscopy (FE-SEM, AURIGA, Carl Zeiss, Germany), and determination of nanofibers diameters were performed with "Image J" software.

\section{Fabrication of SF/CNF Fibrous Composites}

To obtain a homogeneous CNF dispersion, 1 wt.\% CNF solution was sonicated at $300 \mathrm{~W}, 4 \mathrm{~s}$ on/off pulse under the ice/water bath condition. Then, $0-1$ wt.\% of CNF solutions were prepared by dilution with the distilled water. SF fibers (1.6 g) were added to the desired CNF solution and incubated for $10 \mathrm{~min}$ to fully wet the CNF dispersed solution within the SF fibers. The SF/CNF suspension was poured into a metal mold and homogenously distributed. Thereafter, a paper towel was used to remove excessive water from the SF/CNF mixture in the mold (six times). The moisture-free SF/CNF mixture was placed onto a hot press machine (HLP-12H, Han tech, Korea) and the metal mold was removed. Subsequently, the SF/CNF mixture was pressed at $120^{\circ} \mathrm{C}$ for $10 \mathrm{~min}$ to fabricate a SF/CNF fibrous composite. A polyester nonwoven was placed on the top and bottom of the $\mathrm{SF} / \mathrm{CNF}$ mixture to avoid the adhesion of the SF/CNF to the plate of the hot press. The overall process of manufacturing SF/CNF composites is shown in Scheme 1. As a control, untreated nonwoven silk was prepared using the above-mentioned pressing method at room temperature. The resultant SF-CNF composite was labeled SF/CNF-n, where " $n$ " corresponds to the initial CNF content ( $n=5,10$, and $15 \mathrm{wt} . \%$ of SF, respectively).

\section{Characterization}

To determine the CNF distribution on the SF surface, the prepared SF/CNF fibrous composite was immersed in $60 \mathrm{~mL}$ of Direct Red 80 dye solution (0.0025 wt.\%) and incubated for $30 \mathrm{~min}$. Thereafter, distilled water was used to remove the unreacted dye molecules from the sample until no red color was observed on the paper towel, which was pressed onto the SF/CNF fibrous composite sample. The washed fibrous composite was incubated in the oven at $60^{\circ} \mathrm{C}$ for 1 day and images of the dried sample were obtained. To obtain the Direct Red 80-dyed CNF sample, 1 wt.\% of CNF solution was casted on a Petri dish and lyophilized using a freeze dryer. Subsequently, sponge-type CNFs were obtained by pressing at $120^{\circ} \mathrm{C}$ for $10 \mathrm{~min}$. The abovementioned dyeing process was also used for this sample and an image was obtained.

Optical images of the prepared SF/CNF composites were obtained using a digital camera (D7200, Nikon, Japan). The morphologies of the SF/CNF composites were analyzed using field emission scanning electron microscopy (FE-SEM, AURIGA, Carl Zeiss, Germany). To obtain cross-sectional images of the $\mathrm{SF} / \mathrm{CNF}$ composites, the samples were placed in a liquid nitrogen chamber for $6 \mathrm{~h}$ and then cut using a cryo-blade. To find out coating and interfacial adhesion behavior of nanocellulose onto the SF fibers, $1.6 \mathrm{~g}$ of SF fibers were immersed onto the $40 \mathrm{ml}$ of cellulose nanocrystal (CNC) and CNF solution (0.5 wt\%) and it dried $60^{\circ} \mathrm{C}$ for $24 \mathrm{~h}$. The prepared SF/CNC and SF/CNF composites were immersed the $50 \mathrm{ml}$ of distilled water and it sonicated $200 \mathrm{~W}$ for 20 min to detach CNC and CNF from the SF fibers. Then morphology of the SF/CNC and SF/CNF before and after ultrasonication treatment were analyzed using FE-SEM.

The X-ray diffraction (XRD, Rigaku, DMAX 2500) analyses were conducted over a $2 \theta$ range of $5^{\circ}-60^{\circ}$ at $40 \mathrm{kV}$ and $100 \mathrm{~mA}$ using $\mathrm{Cu} \mathrm{K} \alpha$ radiation $(\lambda=0.154 \mathrm{~nm})$. An attenuated total reflectance Fourier transform infrared (ATR-FTIR) spectrometer was used to examine the chemical structures of the SF/CNF composites. The scan range, scan number, and resolution of the measurements were $4000-700 \mathrm{~cm}^{-1}, 128$, and $2 \mathrm{~cm}^{-1}$, respectively. The absolute density $(\rho \mathrm{m})$ of the manufactured SF/CNF composites was measured with a $\mathrm{He}$ pycnometer (AccuPyc 1330, Micromeritics, United States). The samples were weighed and then loaded in the measurement chamber. The envelope density ( $\rho$ e) of the fibrous composites was calculated from the mass and envelope volume of the nonwoven samples. The porosity of the fibrous composite was calculated using Eq. (1):

$$
\text { Porosity }(\%)=\left\{\frac{1-(\text { Enveloped density })}{\text { Absolute Density }}\right\} \times 100
$$

To determine the mechanical properties of the SF/CNF composite, tensile tests were performed using a universal testing machine (CS225, Lloyd Instruments, Ltd., United Kingdom). The experiment was conducted by applying a tensile force in accordance with the main arrangement direction of the SF fibers. The gauge length and width of the samples were fixed to 50 and $25 \mathrm{~mm}$, respectively, and tensile tests were performed using a $1000 \mathrm{~N}$ load cell at an extension rate of 


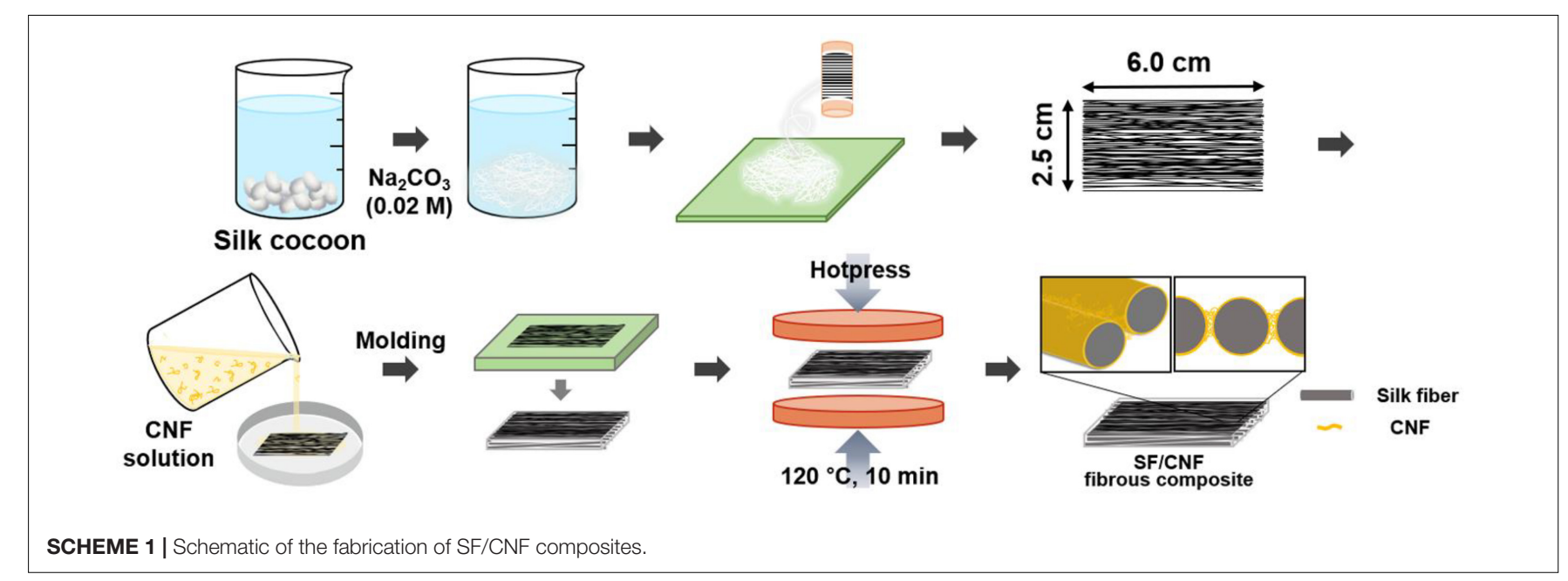

$10 \mathrm{~mm} / \mathrm{min}$. All samples were preconditioned at $20^{\circ} \mathrm{C}$ and a relative humidity of $65 \%$. The Izod impact strength of the unnotched fibrous composite specimens was measured with a (QM700A, Qmesys, Korea) machine at a strike velocity of $2.5 \mathrm{~m} / \mathrm{s}$ according to the ASTMD256 test method. The experiment was conducted by applying an impact force to vertical direction of SF fiber's alignment. At least five samples were tested under each condition and the averages and standard deviations of the tensile strength, elongation at break, and initial Young's modulus were evaluated. To study the physical stability of the SF/CNF composites in a water environment, water droplet images were obtained $1 \mathrm{~s}$ after placing a water droplet on the fibrous composite surface. To investigate the stability under hydrolytic conditions, the SF/CNF composites were immersed in $60 \mathrm{~mL}$ distilled water for 21 days. Subsequently, optical images of the fibrous composites were obtained.

To determine the moisture absorption behavior of the SF/CNF composites, a water uptake test was performed (de Campos et al., 2013). The sample size was fixed to $60 \mathrm{~mm}$ (length) and $25 \mathrm{~mm}$ (width). All samples were preconditioned at $25^{\circ} \mathrm{C}$ and a relative humidity of $51 \%$. Thereafter, the samples were immersed in distilled water. Their weights were determined before and after immersion. At least five samples were tested under each condition and the averages and standard deviations were obtained. The water uptake was calculated using Eq. (2). The parameters $W_{0}$ and $W_{\mathrm{t}}$ are the initial weight and the weight after immersion for $24 \mathrm{~h}$, respectively.

$$
\text { Water uptake }(\%)=\frac{\left(W_{t}-W_{o}\right)}{W_{o}} \times 100
$$

\section{RESULTS AND DISCUSSION}

Using the chemical purification and high intensity ultrasonication process, bamboo-derived cellulose nanofibril (CNF) was successfully prepared. As a result of morphology confirming through the FE-SEM and FE-TEM image, the formation of high aspect ratio CNFs having 15-35 $\mathrm{nm}$ of diameter (average diameter: $26.1 \mathrm{~nm}$ ) was observed (Figure 1).
In order to examine the effect of nonwoven fabrication processing on the silk fibroin (SF) fiber, SF nonwoven fabric was prepared by simply pressing without treatment. Figure 2A shows the image of the degummed SF (sericin removed) and pressed SF nonwoven without heat treatment. The degummed SF shows a three-dimensional cotton candy shape, and it can be confirmed that such SF can be manufactured as a two-dimensional like nonwoven fabric through a simple pressing process. It was found that the pressing process did not change the color of the SF fibers themselves. Meanwhile, images of SF nonwoven and CNF added SF/CNF composite nonwoven prepared by hot pressing method are shown in Figure 2B. In the case of CNF, the hot-pressed CNF film remains white color even though manufactured by the hotpressing method. However, in the case of SF, prepared hotpressed silk non- woven is converted to pale yellow. Generally, a yellowing phenomenon can mainly be observed when protein crosslinking (both self-crosslinking and crosslinking with a chemical crosslinker) occurs. During the fabrication of nonwoven SFs, protein self-crosslinking occurs, resulting in a yellowing phenomenon (Lee et al., 2018). Urreaga and de la Orden (2006) prepared chitosan treated cotton cellulose fibers to examine the interaction between amino group of chitosan and cellulose carbonyl groups and found that the higher the amount of added chitosan, the more conjugated imine groups produced, resulting in yellowing behavior of cellulose. However, it is difficult to observe a significant color difference in the SF/CNF composite compared to the SF heat-treated nonwoven. Therefore, the yellowing phenomenon occurring in the SF/CNF composite is largely affected by the crosslinking of the SF fiber itself.

To confirm the effect of CNF concentration on the residual $\mathrm{CNF}$ contents in the SF/CNF composites, the weight gain ratio of SF/CNF were calculated based on the dry weight and the obtained result was shown in Figure 2C. As shown in the figure, as the CNF content increases to $15 \%$ of the SF weight, the weight gain ratio of the SF/CNF composite also increases proportionally. However, it can be seen that there is a limit to the amount of CNF that can penetrate into the SF nonwoven 

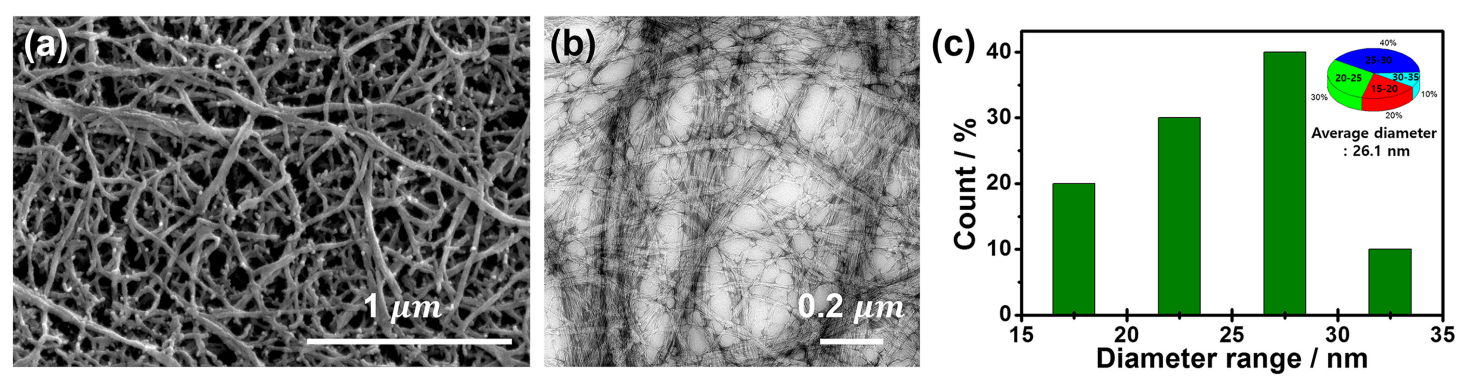

FIGURE 1 | FE-SEM (a), FE-TEM (b) images, and nanofibril diameter distribution (c) of bamboo cellulose nanofibril (CNF)

A

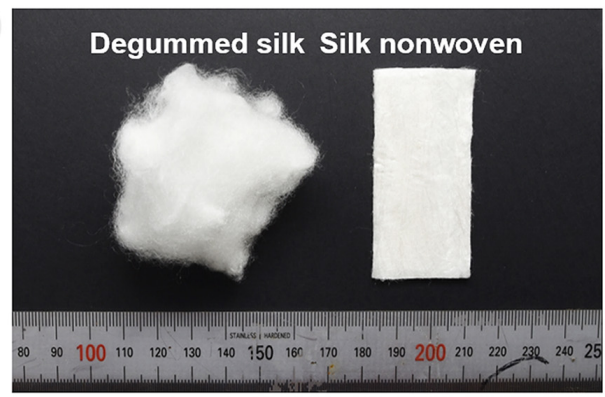

C

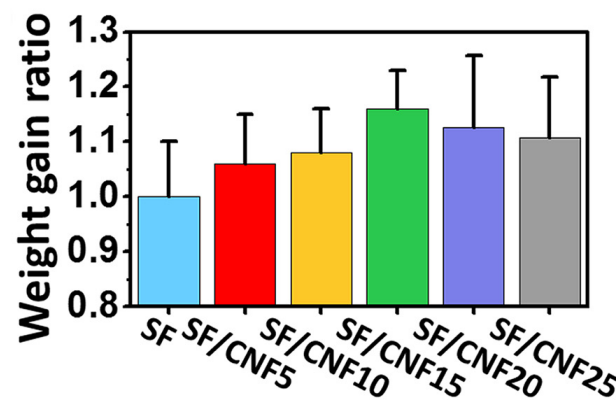

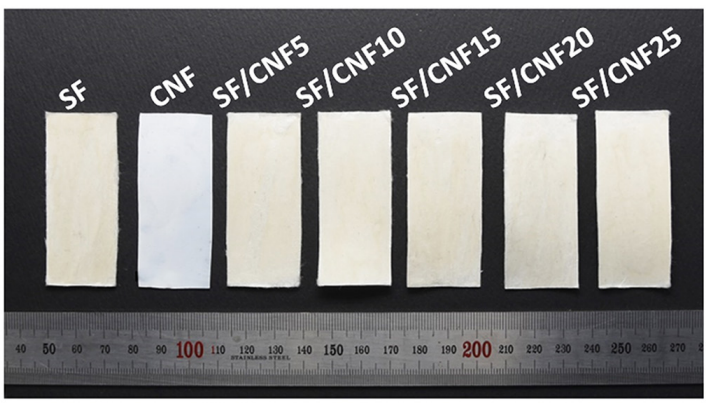

D

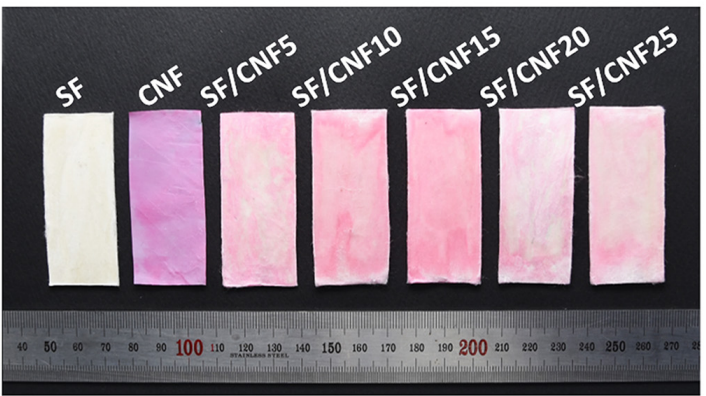

FIGURE 2 | Effect of CNF addition on the visual inspection of SF/CNF composites. Optical images of degummed SF fibers and prepared SF nonwoven without heat treatment (A), hot-pressed SF/CNF composites (B), weight gain ratio of SF/CNF composites (C), and optical images of Direct Red 80 dyed SF/CNF composites (D).

fabric by reduced weight gain ratio of SF/CNF20 and SF/CNF25 compared to SF/CNF15.

In the degumming process, which is the removal of silk sericin from cocoon silk, a Direct Red 80 staining test is widely used to confirm the removal of the gummy sericin protein (Nisal et al., 2014; Kwak et al., 2017). Depend on the hydrophilicity of silk proteins (fibroin and sericin), Direct Red 80 easily stains the gummy and hydrophilic sericin protein, while degummed hydrophobic fibroin fiber cannot be dyed (Kwak and Lee, 2015). In addition, (Saleem et al., 2007) confirmed that the Direct Red 80 dye is easily dyed to cellulose cotton fibers. By using the different dyeability between cellulose and silk fibroin, the distribution of CNF in SF/CNF composite was visualized and the optimal CNF content was found. The Direct Red 80 dyed fibrous SF/CNF composite samples are shown in Figure 2D. As shown in the figure, Direct Red 80 does not dye nonwoven SF, probably because the SFs are hydrophobic and the dye molecules do not penetrate the $\beta$-sheet-dominated SF structure. On the other hand, in the case of the nonwoven CNF fabric, the Direct Red 80 dye penetrates the fabric and a vivid pink color can be observed. This is the result of dyeing through hydrogen bond with cellulose chain after the Direct Red 80 dye dissociates under neutral aqueous conditions. The CNF concentration of the $\mathrm{SF} / \mathrm{CNF}$ composite can be simply determined based on the dyeing performance observed during the Direct Red 80 staining test. As the CNF content of the SF/CNF composites increases from 5 to 15 wt.\%, the color of the fibrous composite surfaces becomes more vivid (pink). In the case of the SF/CNF5 composite, a white part can be observed because the amount of CNF between the SFs is not high enough. In the case of SF/CNF10 and SF/CNF15, the surfaces of the SF fibrous composites are fully covered with CNF molecules, which results in uniform pink layers. In addition, as the CNF weight increases, the color becomes more vivid, indicating that the $\mathrm{CNF}$ concentration of the SF nonwoven is higher. However, SF/CNF20 and SF/CNF25 are partially pale pink similar with SF/CNF5 despite the increase in the CNF 
weight. Resulted concentration effect of CNF may be related to the rheological properties of CNF dispersion. Lasseuguette et al. (2008) investigated the rheological properties of CNF solution according to the concentration. At low or diluted concentration region $(>>0.25 \mathrm{wt} \%)$, the viscosity increased linearly with concentration, whereas at higher concentration the viscosity followed a power law equation. In this study, to consider the water absorption capacity of SF fibers, a higher concentration of $\mathrm{CNF}$ dispersion was used to add more CNF to the SF nonwovens. As a result, as the concentration of CNF increases, the viscosity of the CNF dispersion increases, which makes it difficult for CNF to sufficiently penetrate between the SF fiber surface and the between the SF pores. The penetration tendency of CNF into the SF nonwovens can also be clearly seen in cross sectional images of the SF/CNF composite (Supplementary Figure S1).

To investigate the macroscopic changes of the nonwoven SF structure after the combination of the CNFs with SFs, the envelope and absolute densities of the SF/CNF composites were measured. The porosities of the SF/CNF composites were calculated based on the relationship between the envelope and absolute densities, as shown in Figures $\mathbf{3 A}-\mathbf{C}$ and Supplementary Table S1.

The absolute density $\left(\rho_{\mathrm{m}}\right)$, commonly referred to as bulk density, is the ratio between the mass and the unit volume of a sample. Voids or pores in the material are not considered. The absolute density insignificantly changes, even if CNF is added to the SF structure. On the other hand, the envelope density $\left(\rho_{\mathrm{e}}\right)$, defined as the ratio between the mass of the material and the sample volume, includes voids or pores in the material. As the CNF content increases, the envelope density of the SF/CNF composite increases from $0.79 \pm 0.03$ for nonwoven SF to $1.02 \pm 0.02$ for the SF/CNF15 composite. The calculated porosity was obtained based on the results of two density measurements. The CNF content increases; porosity decreases from $40.91 \pm 1.08$ (sf nonwoven) to $25.91 \pm 1.36$ (SF/CNF15). Kwak et al. (2019a) fabricated jute fiber-sericin preform composites using sericin as bio-adhesive for the adhesion of jute fibers and observed a similar offset phenomenon of the porous structure of the jute fiber composites due to the void filling effect of the sericin proteins. The decrease in the porosity of the SF/CNF composite with increasing $\mathrm{CNF}$ concentration is due to the fact that the CNF does not only form a coating on the SF but also fills the space between the SFs.
To identify the effect of the CNF binder on the morphological changes of the SF/CNF composites, surface and cross-sectional FE-SEM images were obtained. The results are shown in Figure 4. All samples of the SF/CNF composites were found to be well aligned in certain direction even after the hot-pressing process. In the case of SF nonwoven, the SF surface is smooth and the space between the SFs is clearly visible. On the other hand, as CNFs are introduced, the CNF forms a coat on the SFs; at the same time, it fills the space between the SFs. This phenomenon becomes more apparent as the CNF content increases. In the case of the SF/CNF15 composite, CNFs cover the whole SF surface and fill almost all pores between the SFs. This pore filling effect can also be observed in the cross-sectional images (as shown in Figures $\mathbf{4 e - h}$ ). As the CNF content increases, the gaps between the SFs are filled and the sizes of the pores decrease. This effect can be clearly observed in the FE-SEM cross-sectional images with higher magnification. In the case of SF nonwoven, the boundary between the SFs can be clearly observed, whereas in the case of the SF/CNF15 composite, CNFs are observed between SFs, which makes the boundary between fibers unclear. Nevertheless, pores between the SFs can be observed, indicating that the CNFs reduce the porosity of the SF structure but do not fill the entire pore structure of the SF matrix.

To investigate the chemical structure and crystalline properties of SF/CNF composites after CNF addition, FTIR spectra and XRD patterns were obtained. The results are shown in Figure 5. The FTIR spectra of the SF/CNF composites are shown in Figure 5A. In the spectrum of neat CNF, an $\mathrm{O}-\mathrm{H}$ stretching mode at $3300 \mathrm{~cm}^{-1}, \mathrm{C}-\mathrm{H}$ stretching mode at $2800 \mathrm{~cm}^{-1}$, and $\mathrm{C}-\mathrm{O}$ stretching peak at $1013 \mathrm{~cm}^{-1}$ can be observed, which are typically associated with cellulose structures. In the case of SF/CNF composites, the SF shows a crystalline secondary $\beta$-sheet structure typical for SF proteins. Regardless of the CNF content, the spectra of all SF/CNF composites show amide I ( $\mathrm{C}=\mathrm{O}$ stretching) and amide II (N-H bending and C-N stretching) peaks at 1620 and $1510 \mathrm{~cm}^{-1}$, respectively, which are associated with the secondary $\beta$-sheet structure of SF (Jackson and Mantsch, 1995; Lu et al., 2010; Nagarkar et al., 2010; Boulet-Audet et al., 2015). This means that the CNFs do not induce the conformational transition of the $\beta$-sheet structure of $\mathrm{SF}$, even if CNFs are introduced to the SF structure. In general, silk-based materials, including natural and regenerated SFs, have excellent mechanical properties when the silk materials exhibit a $\beta$-sheet structure. Therefore, maintaining the secondary
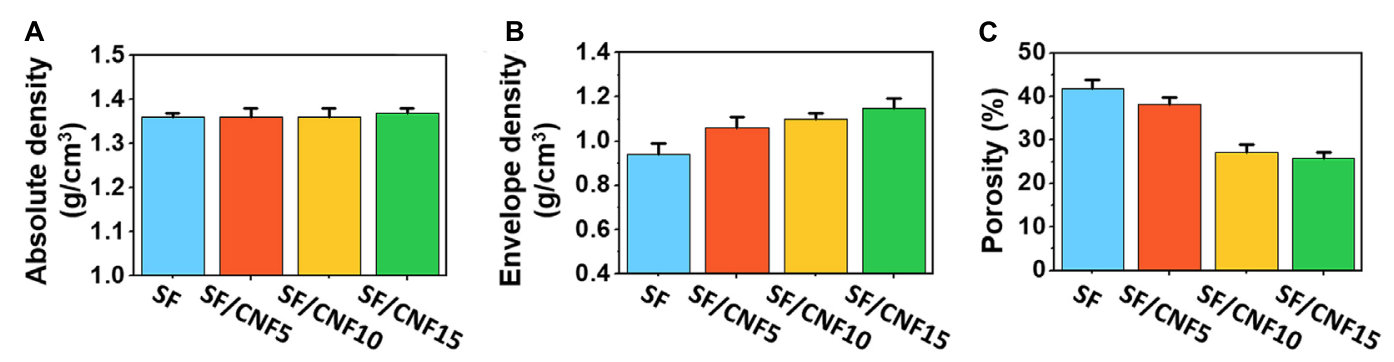

FIGURE 3 | Absolute density (A), envelope density (B), and calculated porosity (C) of the SF/CNF composites with various amounts of CNFs. 

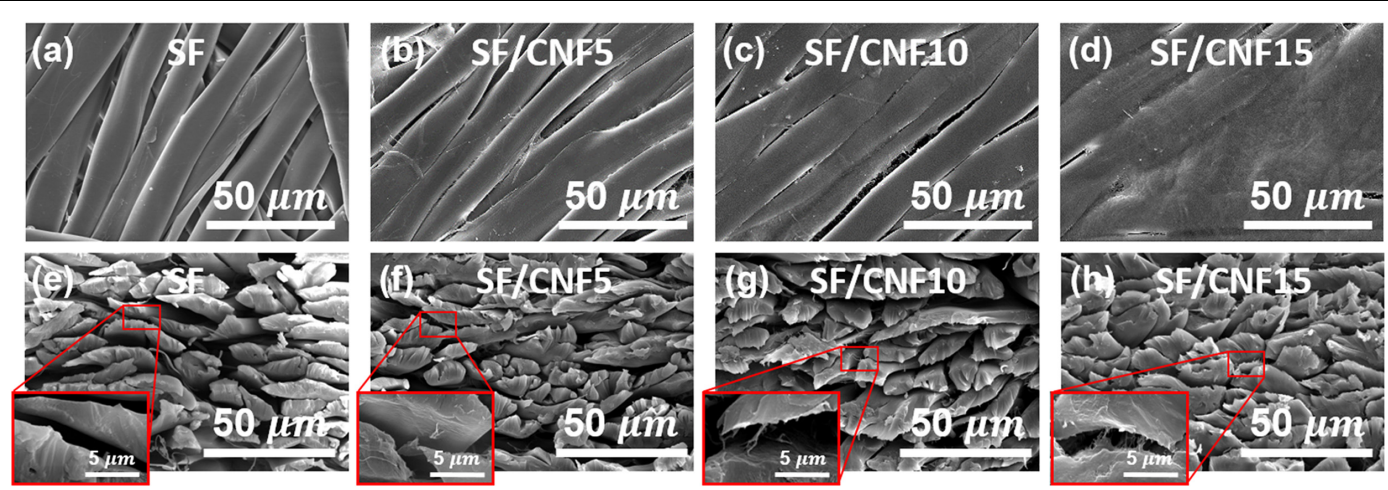

FIGURE 4 | FE-SEM images of the SF/CNF composites with various CNF concentrations. Surface (a-d) and cross section (e-h) images; (a,e): SF nonwoven, $(\mathbf{b}, \mathbf{f}):$ SF/CNF5, (c,g): SF/CNF10, (d,h): SF/CNF15. The insets represent high-magnitude images.
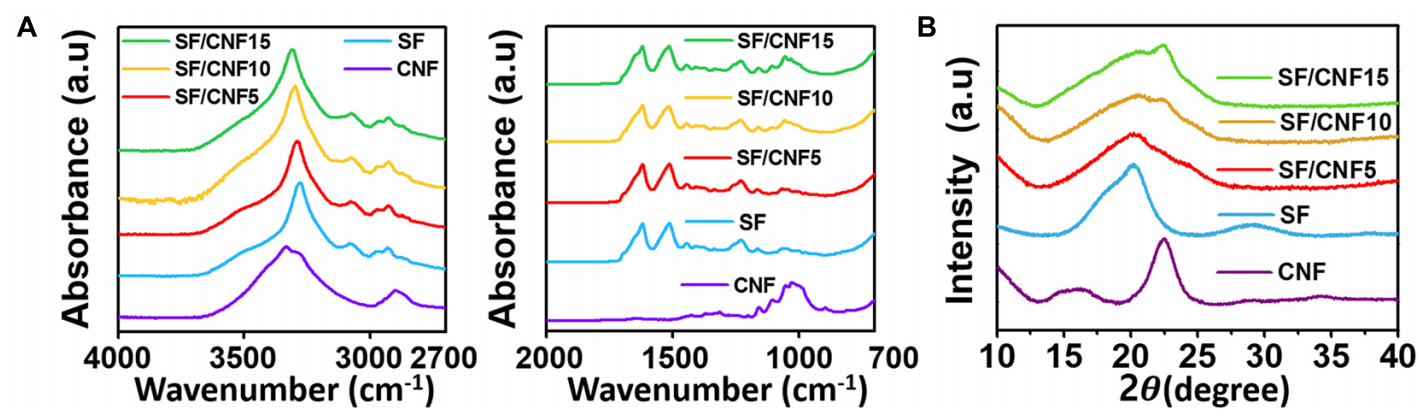

FIGURE 5 | Characteristics of CNF, nonwoven SF, and SF/CNF composites with various amounts of CNFs: (A) FTIR spectra, (B) XRD pattern.

$\beta$-sheet structure of the SF, even when CNF is added, will have a positive effect on the mechanical properties. On the other hand, as the CNF content increases, the intensity of the C-O stretching absorption peak at $1013 \mathrm{~cm}^{-1}$ increases, (Morán et al., 2008; Han et al., 2013; Feng et al., 2019) implying the settling of CNF on the surface of the SF. Furthermore, in the spectra of the SF/CNF composites, the O-H peak of SF merges with that of CNF. The spectra exhibit a blue-shifted broader peak, indicating the interaction between SFs and CNFs through the hydrogen bonds (Lee et al., 2013).

The XRD patterns of the SF/CNF composites are shown in Figure 5B. The nonwoven SF exhibits a peak at $20.2^{\circ}$, which corresponds to the silk II $\beta$-sheet crystallite structure (Lee, 2004; Cheng et al., 2015; Guang et al., 2015; Ming et al., 2015). In the case of CNF, characteristic peaks at $2 \theta=16.3^{\circ}$ and $22.6^{\circ}$ are observed, representative for the typical cellulose type I structure (Morán et al., 2008; Han et al., 2013; Kwak et al., 2019b). Based on the XRD patterns of the SF/CNF composites, the CNF binder affects their crystalline structures. In the case of SF/CNF5, because the CNF does not cover the whole SF surface, the crystal structure of SF appears to be sharp. On the other hand, in the case of SF/CNF10, the crystal structure appears to be mixed, but the peak at $20.2^{\circ}$, which represents the crystal structure of silk, is more dominant. In the case of SF/CNF15 with a higher CNF content, the peak at $22.6^{\circ}$, which corresponds to CNF, is more prevalent. Based on the FTIR and XRD results, the introduction of CNF does not lead to the transfer of the $\beta$-sheet structure of SF, but the crystal structure of the entire SF/CNF composite changes.

To visually identify the interaction between CNF and SF, a ultrasonication detaching test was applied using nanocellulose coated SF fibers. To find out the effect of nanocellulose morphology on the interaction between SF and nanocellulose, cellulose nanocrystal (CNC) with a low aspect ratio and CNF with a high aspect ratio were used as a SF coating agent. Thereafter, bonded nanocellulose onto SF fibers were detached by ultrasonic treatment, which is a strong physical treatment technique. Figure 6a shows the morphology of a freeze-dried $\mathrm{CNC}$. It can be seen that the aspect ratio of $\mathrm{CNC}$ is lower than that of CNF as shown above image (Figure 1a). Also, as shown in Figure 6b, neat silk fibers have a smooth surface. Figure 6d show that the SF has a smooth surface, while the CNC-coated SF has a rough surface. This smooth surface of SF changed to rough with a short aspect ratio of CNC covering (Figure 6c). The rough surface of the CNC-coated SF indicates that the $\mathrm{CNC}$ covers the surface of the SF and forms a core-shell-like structure. Although the roughness decreases after sonication, the CNC-coated SF surface is rougher than that of the conventional SFs. This means that more than just physical adsorption occurs between $\mathrm{CNC}$ and SF. Compared with $\mathrm{CNC}, \mathrm{CNF}$ has a longer aspect ratio, so it can be applied not only for simple coating but also for interfacial adhesion between SF fibers. In order to confirm the adhesion behavior of CNF, CNF coating was applied 
to SF fibers, and FE-SEM images of SF/CNF fibers subjected to sonication to separate them are shown in Figures $\mathbf{6 e , f}$. As can be seen in the figure, the CNF bundle wraps around the SF fibers, so the SF fibers have a rough surface. Not only this, you can clearly observe the presence of CNF bundles between SF fibers. Through this, when the CNF is introduced onto the SF fibers, CNF can play not only the simple coating reagent but also the interfacial adhesive of the SF fibers. On the other hand, similar to the CNC, defibrillation and detachment of CNF from the SF fibers occurred by strong ultrasonication treatment, but nanoscaled CNF bundles exist, indicating that there is a certain binding force between CNF and SF. However, further consideration of the exact mechanism between CNF and SF is needed.

Based on the introduction of CNF, which allows the simultaneous coating and binding of SFs, SF/CNF composites with excellent mechanical properties can be easily fabricated. To confirm this effect, the mechanical properties of SF/CNF composites with various amounts of CNFs were investigated using impact and tensile tests. The results are shown in Figure 7 and Supplementary Table S2. Figures 7A,B present the average tensile strength and modulus of SF/CNF composites with the various amounts of CNFs, respectively, and indicate the improved tensile behavior of the SF/CNF composites. The
SF/CNF fibrous composite with 5 wt.\% CNF (SF/CNF5) exhibits a 60 and $61 \%$ increase in the tensile strength and modulus, respectively, compared with nonwoven SF. The mechanical reinforcement further enhances with increasing CNF content. The SF/CNF 15 composite shows a $110 \%$ and $150 \%$ increase in the tensile strength and modulus, respectively. The impact strength test results (Figure 7C) confirm the mechanical reinforcement effect of CNF. Based on the impact tests, the SF/CNF5 displays a $\sim 53 \%$ increase in the impact strength compared with SF nonwoven, whereas SF/CNF10 and SF/CNF15 show an increase in the impact strength of $\sim 109$ and $\sim 228 \%$, respectively. These data prove that $\mathrm{CNF}$ can reinforce the low impact strength of nonwoven SF and that higher CNF concentrations can accentuate this trend. The mechanical reinforcement phenomenon of SF/CNF composites can be explained as follows. First, because the introduction of CNF does not affect the secondary $\beta$-sheet structure of SF, the mechanical properties of the SFs can be maintained, even after the preparation of the SF/CNF composites. In addition, by coating CNF onto the SF surface, a core-shell-structured SF/CNF fiber forms, which promotes the hydrogen bonding between the microscale SFs and nanoscale CNFs. In addition, as the amount of CNF increases, high-aspect ratio CNFs can form an interfibrillar bridges between the hybrid fibers of the SF/CNF composites,
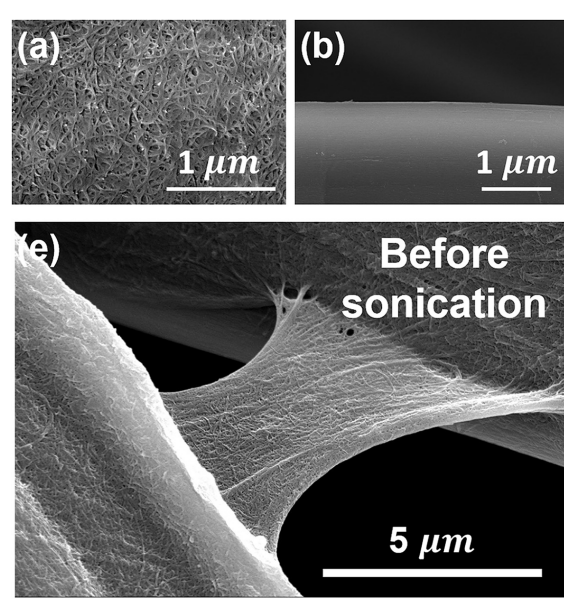
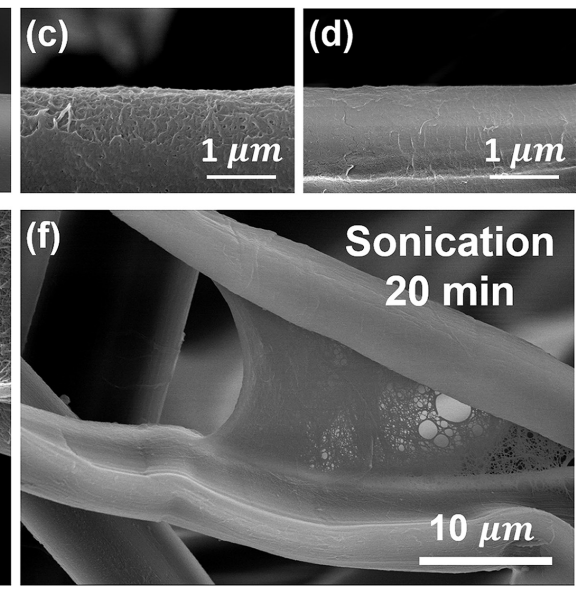

FIGURE 6 | FE-SEM image of (a) neat CNC, (b) neat silk, SF/CNC, SF/CNF before (c,e) and after (d,f) sonication treatment for 20 min.
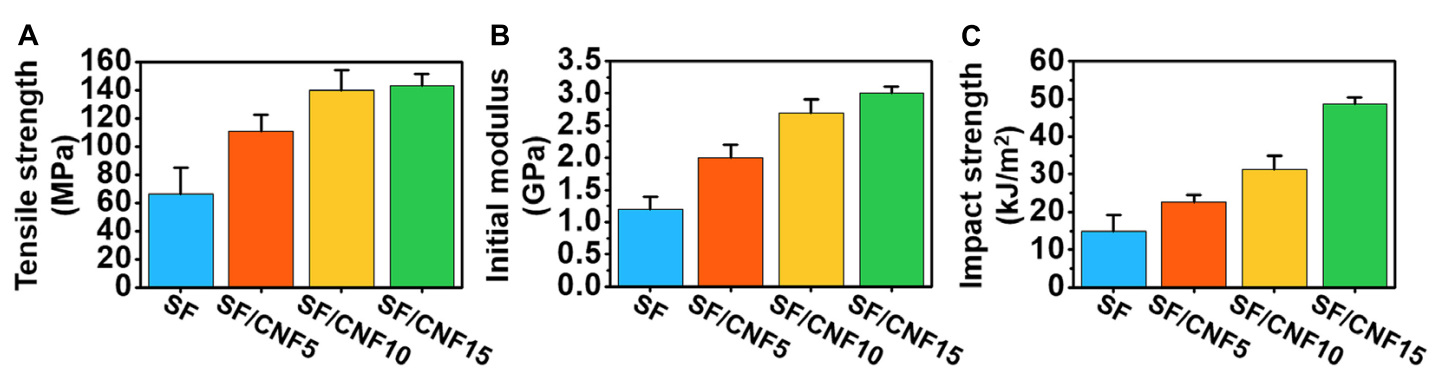

FIGURE 7 | Mechanical properties of nonwoven SF and SF/CNF composites with various amounts of CNFs: (A) tensile strength, (B) tensile modulus, and (C) impact strength. 
A
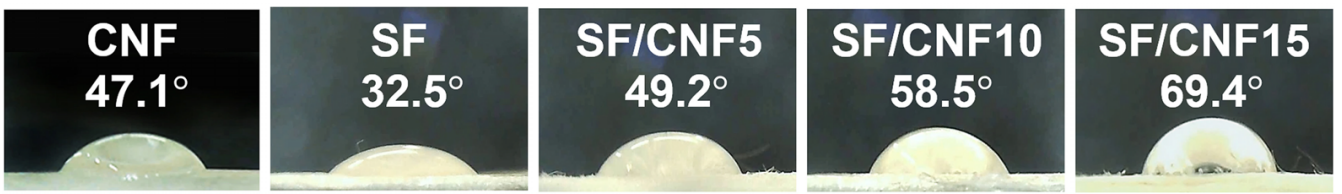

B

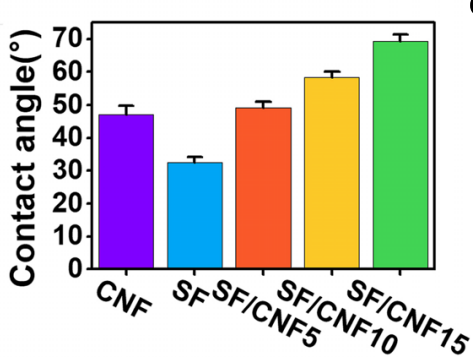

C

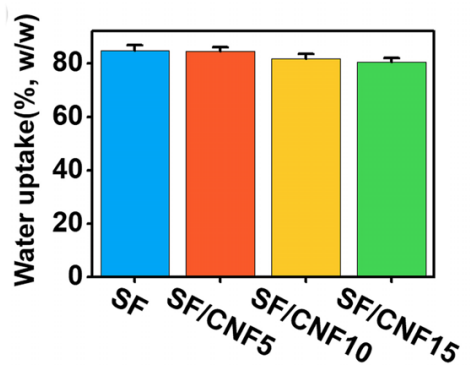

D

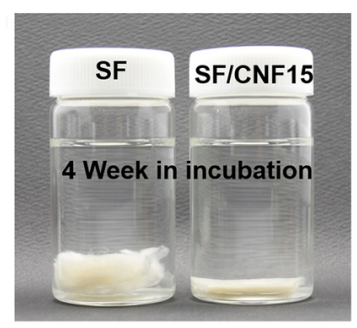

FIGURE 8 | (A,B) Initial water contact angles of the SF/CNF composites with various amounts of CNFS, (C) water uptake of SF/CNF composites with various amounts of CNFs after $24 \mathrm{~h}$, and (D) nonwoven SF and SF/CNF15 composite after 4 weeks of incubation.

thereby improving the interfacial adhesion characteristics of the SF/CNF composites.

The stability of the fibrous structure including the nonwoven fabric in wet or high-humidity environments is a very important property. Because most of the natural fibers are hydrophilic, composite materials with natural fibers as reinforcing agents often have disadvantages in that they are more vulnerable to moisture environments than materials without a reinforced matrix (Fages et al., 2013; Theng et al., 2015, 2017). Many natural polymer-based CNF-reinforced composites fabricated using common blending methods show hydrophilic properties such as a decrease in the water contact angle (WCA) (Kwak et al., 2018; Wang et al., 2018a), increasing water uptake or absorption capacity, and higher swelling ratio with increasing CNF concentration. On the other hand, when the surface coating and layer-by-layer coating method are used, the CNF-reinforced composite materials have hydrophobic properties because CNF actively affects the surface morphologies or topologies of the composite materials. The WCA is used as an indicator for the degree of surface hydrophobicity or hydrophilicity of film, textile, and membrane materials. Water droplet images representative of the SF/CNF fibrous composites and the average WCA results are shown in Figures 8A,B. Generally, a film with a WCA above $65^{\circ}$ is considered as hydrophobic (Vogler, 1998). The WCA of the nonwoven SF without CNF is $\sim 32.5^{\circ}$, which indicates that the nonwoven SF is hydrophilic. In contrast, when $\mathrm{CNF}$ is added to the SF structure, the WCA significantly increases. In the case of SF/CNF15, the contact angle is $\sim 69.4^{\circ}$, which means that the CNF coating on the SF surface and CNF bonding with SFs sufficiently hydrophobizes the surfaces of the SF/CNF composites. The hydrophobic effect of CNF is due to the change in the surface topology of the entire SF/CNF composite.

In addition, the hydrostability of SF/CNF composites in wet environments was also examined using a water uptake test. The results are shown in Figure 8C. As the CNF content increases, the water uptake of the SF/CNF composites decreases
(Fages et al., 2013; Theng et al., 2015, 2017). This might have the following reasons: (1) the initial diffusion rate of the water molecules decreases because of the aforementioned surface hydrophobicity, and (2) by introducing CNF into SF fibrous systems, the porosity of the SF/CNF composites decreases, thus reducing the space for water impregnation. Finally, the increase in the hydrogen bonding between SF$\mathrm{CNF}$ and $\mathrm{CNF}-\mathrm{CNF}$ reduces the affinity to water molecules. The hydrophobic effect of CNF greatly contributes to the morphological stability of the SF/CNF composites in wet environments. As shown in Figure 8D, nonwoven SF without CNF shows that each SF disintegrates in a 4-week stirring environment, causing the loss of the dense SF structure. In contrast, the dense nonwoven structure of the SF/CNF15 composite can be well maintained, even after 4 weeks of stirring in an aqueous environment.

\section{CONCLUSION}

In this study, natural micro- and nano-fiber fused fibrous composites were fabricated using silk and cellulose as green raw materials. Robust SF/CNF composites were fabricated using a simple short-term thermal bonding process. This process is eco-friendly and energy-efficient because it does not require an organic solvent, additional chemical reagents, long processing time, and high energy consumption. The introduction of CNF to SF increases the interfibrillar bonding of SFs as well as the coating on the SF surface. As the CNF content increases, the coating and bonding effects of CNF rapidly increase. Therefore, the mechanical properties and structural stability of the SF/CNF composites increase compared with that of nonwoven SF without CNF. A maximum of 15 wt.\% CNF can be added to the SF/CNF composite. The SF/CNF15 composite shows a 110 and $225 \%$ increase in the impact and tensile strength, respectively, compared with nonwoven SF. The results also show that the hydrophobicity of the SF/CNF composite 
surface and bulk materials can be imparted. Such green and facile $\mathrm{SF} / \mathrm{CNF}$ composites have immense potential for a wide range of applications, particularly in the nonwoven and fiber-reinforced plastics industries.

\section{DATA AVAILABILITY STATEMENT}

All datasets generated for this study are included in the article/Supplementary Material.

\section{AUTHOR CONTRIBUTIONS}

The manuscript was written through contributions of all authors. All authors have given approval to the final version of the manuscript.

\section{REFERENCES}

Adekunle, K. F. (2015). Surface treatments of natural fibres-a review: part 1. OJPChem. 05, 41-46. doi: 10.4236/ojpchem.2015.53005

Amini, E., Tajvidi, M., Gardner, D. J., and Bousfield, D. W. (2017). Utilization of cellulose nanofibrils as a binder for particleboard manufacture. Bio Resour. 12, 4093-4110. doi: 10.15376/biores.12.2.4093-4110

Arévalo, R., and Peijs, T. (2016). Binderless all-cellulose fibreboard from microfibrillated lignocellulosic natural fibres. Compos. Part A Appl. Sci. Manuf. 83, 38-46. doi: 10.1016/j.compositesa.2015.11.027

Asakura, T., Okushita, K., and Williamson, M. P. (2015). Analysis of the Structure of Bombyx mori silk fibroin by NMR. Macromolecules 48, 2345-2357. doi: 10.1021/acs.macromol.5b00160

Baiardo, M., Zini, E., and Scandola, M. (2004). Flax fibre-polyester composites. Compos. A Appl. Sci. Manuf. 35, 703-710. doi: 10.1016/j.compositesa.2004. 02.004

Boulet-Audet, M., Vollrath, F., and Holland, C. (2015). Identification and classification of silks using infrared spectroscopy. J. Exp. Biol. 218, 3138-3149. doi: $10.1242 /$ jeb. 128306

Carvalho, K. C. C., Mulinari, D. R., Voorwald, H. J. C., and Cioffi, M. O. H. (2010). Chemical modification effect on the mechanical properties of hips/ coconut fiber composites. Bio Resour. 5, 1143-1155.

Cervin, N. T., Andersson, L., Ng, J. B. S., Olin, P., Bergström, L., and Wågberg, L. (2013). Lightweight and strong cellulose materials made from aqueous foams stabilized by nanofibrillated cellulose. Biomacromolecules 14, 503-511. doi: 10 . 1021/bm301755u

Chen, F., Porter, D., and Vollrath, F. (2012). Structure and physical properties of silkworm cocoons. J. R. Soc. Interface 9, 2299-2308. doi: 10.1098/rsif.2011.0887

Cheng, G., Wang, X., Tao, S., Xia, J., and Xu, S. (2015). Differences in regenerated silk fibroin prepared with different solvent systems: from structures to conformational changes. J. Appl. Polym. Sci. 132, 1-8. doi: 10.1002/app.41959

Cho, H. J., Yoo, Y. J., Kim, J. W., Park, Y. H., Bae, D. G., and Um, I. C. (2012). Effect of molecular weight and storage time on the wet- and electro-spinning of regenerated silk fibroin. Polym. Degrad. Stab. 97, 1060-1066. doi: 10.1016/j. polymdegradstab.2012.03.007

Dányádi, L., Móczó, J., and Pukánszky, B. (2010). Effect of various surface modifications of wood flour on the properties of PP/wood composites. Compos. Part A Appl. Sci. Manuf. 41, 199-206. doi: 10.1016/j.compositesa.2009.10.008

Das, O., Sarmah, A. K., and Bhattacharyya, D. (2015). A sustainable and resilient approach through biochar addition in wood polymer composites. Sci. Total Environ. 51, 326-336. doi: 10.1016/j.scitotenv.2015.01.063

de Campos, A., Tonoli, G. H. D., Marconcini, J. M., Mattoso, L. H. C., Klamczynski, A., Gregorski, K. S., et al. (2013). TPS/PCL composite reinforced with treated sisal fibers: property. J. Polym. Environ. 21, 1-7. doi: 10.1007/s10924-0120512-8

\section{FUNDING}

This work was supported by the National Research Foundation of Korea (NRF) grant funded by the Korea government (MSIT) (NRF-2020R1C1C1012623), by Basic Science Research Program through the National Research Foundation of Korea (NRF) funded by the Ministry of Education (NRF2019R1A2B5B01070270), and by a grant from the National Institute of Fisheries Science, South Korea (R2020038).

\section{SUPPLEMENTARY MATERIAL}

The Supplementary Material for this article can be found online at: https://www.frontiersin.org/articles/10.3389/fmats. 2020.00098/full\#supplementary-material

Duan, B., Shou, K., Su, X., Niu, Y., Zheng, G., Huang, Y., et al. (2017). Hierarchical microspheres constructed from chitin nanofibers penetrated hydroxyapatite crystals for bone regeneration. Biomacromolecules 18, 2080-2089. doi: 10.1021/ acs.biomac.7b00408

Eichhorn, S. J., Dufresne, A., Aranguren, M., Marcovich, N. E., Capadona, J. R., Rowan, S. J., et al. (2010). Review: current international research into cellulose nanofibres and nanocomposites. J. Mater Sci. 45, 1-33. doi: 10.1007/s10853009-3874-0

Fages, E., Cano, M., Gironés, S., Boronat, T., Fenollar, O., and Balart, R. (2013). The use of wet-laid techniques to obtain flax nonwovens with different thermoplastic binding fibers for technical insulation applications. Textile Res. J. 83, 426-437. doi: 10.1177/0040517512454183

Favaro, S. L., Ganzerli, T. A., de Carvalho Neto, A. G. V., da Silva, O. R. R. F., and Radovanovic, E. (2010). Chemical, morphological and mechanical analysis of sisal fiber-reinforced recycled high-density polyethylene composites. Express Polym. Lett. 4, 465-473. doi: 10.3144/expresspolymlett.2010.59

Feng, Y., Li, X., Zhang, Q., Ye, D., Li, M., You, R., et al. (2019). Fabrication of porous silk fibroin/cellulose nanofibril sponges with hierarchical structure using a lithium bromide solvent system. Cellulose 26, 1013-1023. doi: 10.1007/ s10570-018-2149-9

Fortea-Verdejo, M., Lee, K.-Y., Zimmermann, T., and Bismarck, A. (2016). Upgrading flax nonwovens: nanocellulose as binder to produce rigid and robust flax fibre preforms. Compos. Part A App. Sci. Manuf. 83, 63-71. doi: 10.1016/j. compositesa.2015.11.021

Gao, Y., Hou, M., Yang, R., Zhang, L., Xu, Z., Kang, Y., et al. (2019). Highly porous silk fibroin scaffold packed in PEGDA/sucrose microneedles for controllable transdermal drug delivery. Biomacromolecules 20, 1334-1345. doi: 10.1021/acs. biomac. 8 b01715

Guang, S., An, Y., Ke, F., Zhao, D., Shen, Y., and Xu, H. (2015). Chitosan/silk fibroin composite scaffolds for wound dressing. J. Appl. Polym. Sci. 132, 1-7. doi: 10.1002/app.42503

Guo, F., Wang, N., Cheng, Q., Hou, L., Liu, J., Yu, Y., et al. (2016). Low-cost coir fiber composite with integrated strength and toughness. ACS Sustainable Chem. Eng. 4, 5450-5455. doi: 10.1021/acssuschemeng.6b00830

Hadadi, A., Whittaker, J. W., Verrill, D. E., Hu, X., Larini, L., and Salas-de la Cruz, D. (2018). A hierarchical model to understand the processing of polysaccharides/protein-based films in ionic liquids. Biomacromolecules 19, 3970-3982. doi: 10.1021/acs.biomac.8b00903

Han, C., Yao, Y., Cheng, X., Luo, J., Luo, P., Wang, Q., et al. (2017). Electrophoretic deposition of gentamicin-loaded silk fibroin coatings on $3 \mathrm{~d}$ printed porous cobalt-chromium-molybdenum bone substitutes to prevent orthopedic implant infections. Biomacromolecules 18, 3776-3787. doi: 10.1021/ acs.biomac.7b01091

Han, J., Zhou, C., Wu, Y., Liu, F., and Wu, Q. (2013). Self-assembling behavior of cellulose nanoparticles during freeze-drying: effect of 
suspension concentration, particle size, crystal structure, and surface charge. Biomacromolecules 14, 1529-1540. doi: 10.1021/bm4001734

Jackson, M., and Mantsch, H. H. (1995). The use and misuse of ftir spectroscopy in the determination of protein structure. Crit. Rev. Biochem. Mol. Biol. 30, 95-120. doi: 10.3109/10409239509085140

Juntaro, J., Pommet, M., Kalinka, G., Mantalaris, A., Shaffer, M. S. P., and Bismarck, A. (2008). Creating hierarchical structures in renewable composites by attaching bacterial cellulose onto sisal fibers. Adv. Mater. 20, 3122-3126. doi: 10.1002/adma.200703176

Keten, S., Xu, Z., Ihle, B., and Buehler, M. J. (2010). Nanoconfinement controls stiffness, strength and mechanical toughness of $\beta$-sheet crystals in silk. Nat. Mater 9, 359-367. doi: 10.1038/nmat2704

Kim, H.-J., Jeong, Y.-H., Choe, H.-C., and Brantley, W. A. (2014). Hydroxyapatite formation on biomedical $\mathrm{Ti}-\mathrm{Ta}-\mathrm{Zr}$ alloys by magnetron sputtering and electrochemical deposition. Thin Solid Films 572, 119-125. doi: 10.1016/j.tsf. 2014.07.058

Klemm, D., Heublein, B., Fink, H.-P., and Bohn, A. (2005). Cellulose: fascinating biopolymer and sustainable raw material. Angew. Chem. Int. Ed. 44, 3358-3393. doi: 10.1002/anie.200460587

Klemm, D., Kramer, F., Moritz, S., Lindström, T., Ankerfors, M., Gray, D., et al. (2011). Nanocelluloses: a new family of nature-based materials. Angew. Chem. Int. Ed. 50, 5438-5466. doi: 10.1002/anie.201001273

Koh, L.-D., Cheng, Y., Teng, C.-P., Khin, Y.-W., Loh, X.-J., Tee, S.-Y., et al. (2015). Structures, mechanical properties and applications of silk fibroin materials. Prog. Polym. Sci. 46, 86-110. doi: 10.1016/j.progpolymsci.2015.02.001

Kwak, H. W., Eom, J., Cho, S. Y., Lee, M. E., and Jin, H.-J. (2019a). Hightoughness natural polymer nonwoven preforms inspired by silkworm cocoon structure. Int. J. Biol. Macromol. 127, 146-152. doi: 10.1016/j.ijbiomac.2019. 01.005

Kwak, H. W., You, J., Lee, M. E., and Jin, H.-J. (2019b). Prevention of cellulose nanofibril agglomeration during dehydration and enhancement of redispersibility by hydrophilic gelatin. Cellulose 26, 4357-4369. doi: 10.1007/ s10570-019-02387-z

Kwak, H. W., Ju, J. E., Shin, M., Holland, C., and Lee, K. H. (2017). Sericin promotes fibroin silk i stabilization across a phase-separation. Biomacromolecules 18, 2343-2349. doi: 10.1021/acs.biomac.7b00549

Kwak, H. W., Lee, H., Lee, M. E., and Jin, H.-J. (2018). Facile and green fabrication of silk sericin films reinforced with bamboo-derived cellulose nanofibrils. J. Cleaner Prod. 200, 1034-1042. doi: 10.1016/j.jclepro.2018.07.289

Kwak, H. W., and Lee, K. H. (2015). Monitoring of phase separation between silk fibroin and sericin using various dye system. Int. J. Ind. Entomol. 30, 1-5. doi: 10.7852/ijie.2015.30.1.1

Lasseuguette, E., Roux, D., and Nishiyama, Y. (2008). Rheological properties of microfibrillar suspension of TEMPO-oxidized pulp. Cellulose 15, 425-433. doi: 10.1007/s10570-007-9184-2

Lee, J., Le Boulicaut, N., Kwon, O. H., Park, W. H., and Cho, D. (2019). Electron beam irradiation effect on the mechanical and thermal properties of 2-D silk fibroin fabric/poly(lactic acid) biocomposites. J. Ind. Eng. Chem. 71, 150-159. doi: 10.1016/j.jiec.2018.11.017

Lee, J. H., Bae, C. H., Park, B.-D., and Um, I. C. (2013). Preparation of cellulose nanofibril/regenerated silk fibroin composite fibers. Int. J. Ind. Entomol. 26, 81-88. doi: 10.7852/IJIE.2013.26.2.81

Lee, J. H., Bae, Y. S., Kim, S. J., Song, D. W., Park, Y. H., Bae, D. G., et al. (2018). Preparation of new natural silk non-woven fabrics by using adhesion characteristics of sericin and their characterization. Int. J. Biol. Macromol. 106, 39-47. doi: 10.1016/j.ijbiomac.2017.07.179

Lee, K. H. (2004). Silk sericin retards the crystallization of silk fibroin. Macromol. Rapid Commun. 25, 1792-1796. doi: 10.1002/marc.20040 0333

Lee, K.-Y., Bharadia, P., Blaker, J. J., and Bismarck, A. (2012). Short sisal fibre reinforced bacterial cellulose polylactide nanocomposites using hairy sisal fibres as reinforcement. Compos. Part A Appl. Sci. Manuf. 43, 2065-2074. doi: 10.1016/ j.compositesa.2012.06.013

Lin, T.-Y., Masunaga, H., Sato, R., Malay, A. D., Toyooka, K., Hikima, T., et al. (2017). Liquid crystalline granules align in a hierarchical structure to produce spider dragline microfibrils. Biomacromolecules 18, 1350-1355. doi: 10.1021/ acs.biomac. 7 b00086
Lu, Q., Hu, X., Wang, X., Kluge, J. A., Lu, S., Cebe, P., et al. (2010). Waterinsoluble silk films with silk I structure. Acta Biomaterialia 6, 1380-1387. doi: 10.1016/j.actbio.2009.10.041

Magaz, A., Roberts, A. D., Faraji, S., Nascimento, T. R. L., Medeiros, E. S., Zhang, W., et al. (2018). Porous, aligned, and biomimetic fibers of regenerated silk fibroin produced by solution blow spinning. Biomacromolecules 19, 4542-4553. doi: 10.1021/acs.biomac.8b01233

Malay, A. D., Sato, R., Yazawa, K., Watanabe, H., Ifuku, N., Masunaga, H., et al. (2016). Relationships between physical properties and sequence in silkworm silks. Sci. Rep. 6:27573. doi: 10.1038/srep27573

Ming, J., Pan, F., and Zuo, B. (2015). Influence factors analysis on the formation of silk I structure. Int. J. Biol. Macromol. 75, 398-401. doi: 10.1016/j.ijbiomac. 2015.02.002

Morán, J. I., Alvarez, V. A., Cyras, V. P., and Vázquez, A. (2008). Extraction of cellulose and preparation of nanocellulose from sisal fibers. Cellulose 15, 149-159. doi: 10.1007/s10570-007-9145-9

Mozumder, M. S., Zhang, H., and Zhu, J. (2011). Mimicking lotus leaf: development of micro-nanostructured biomimetic superhydrophobic polymeric surfaces by ultrafine powder coating technology. Macromol. Mater. Eng. 296, 929-936. doi: 10.1002/mame.201100080

Nagarkar, S., Nicolai, T., Chassenieux, C., and Lele, A. (2010). Structure and gelation mechanism of silk hydrogels. Phys. Chem. Chem. Phys. 12:3834. doi: 10.1039/b916319k

Nisal, A., Trivedy, K., Mohammad, H., Panneri, S., Sen Gupta, S., Lele, A., et al. (2014). Uptake of azo dyes into silk glands for production of colored silk cocoons using a green feeding approach. ACS Sustainable Chem. Eng. 2, 312-317. doi: $10.1021 /$ sc400355k

Numata, K., Yamazaki, S., and Naga, N. (2012). Biocompatible and biodegradable dual-drug release system based on silk hydrogel containing silk nanoparticles. Biomacromolecules 13, 1383-1389. doi: 10.1021/bm300089a

Omenetto, F. G., and Kaplan, D. L. (2010). New Opportunities For An Ancient Material. Science 329, 528-531. doi: 10.1126/science.1188936

Pandey, A., Jalihal, D., Kalra, D., Mn, D., and Prabhu, D. (2015). Estimation of adiponectin levels in diabetic, non-diabetic fatty liver diseases and healthy controls. Int. J. Res. Med. Sci. 3, 140-146. doi: 10.5455/2320-6012. ijrms20150124

Peng, Z., Yang, X., Liu, C., Dong, Z., Wang, F., Wang, X., et al. (2019). Structural and Mechanical properties of silk from different instars of Bombyx mori. Biomacromolecules 20, 1203-1216. doi: 10.1021/acs.biomac.8b01576

Pommet, M., Juntaro, J., Heng, J. Y. Y., Mantalaris, A., Lee, A. F., Wilson, K., et al. (2008). Surface modification of natural fibers using bacteria: depositing bacterial cellulose onto natural fibers to create hierarchical fiber reinforced nanocomposites. Biomacromolecules 9, 1643-1651. doi: 10.1021/bm800169g

Saba, N., Mohammad, F., Pervaiz, M., Jawaid, M., Alothman, O. Y., and Sain, M. (2017a). Mechanical, morphological and structural properties of cellulose nanofibers reinforced epoxy composites. Int. J. Biol. Macromol. 97, 190-200. doi: 10.1016/j.ijbiomac.2017.01.029

Saba, N., Safwan, A., Sanyang, M. L., Mohammad, F., Pervaiz, M., Jawaid, M., et al. (2017b). Thermal and dynamic mechanical properties of cellulose nanofibers reinforced epoxy composites. Int. J. Biol. Macromol. 102, 822-828. doi: 10.1016/ j.ijbiomac.2017.04.074

Saleem, M., Pirzada, T., and Qadeer, R. (2007). Sorption of acid violet 17 and direct red 80 dyes on cotton fiber from aqueous solutions. Colloid Surf. APhysicochem. Eng. Asp. 292, 246-250. doi: 10.1016/j.colsurfa.2006.06.035

Sanjay, M. R., Madhu, P., Jawaid, M., Senthamaraikannan, P., Senthil, S., and Pradeep, S. (2018). Characterization and properties of natural fiber polymer composites: a comprehensive review. J. Cleaner Prod. 172, 566-581. doi: 10. 1016/j.jclepro.2017.10.101

Shah, D. U., Porter, D., and Vollrath, F. (2014). Can silk become an effective reinforcing fibre? A property comparison with flax and glass reinforced composites. Compos. Sci. Technol. 101, 173-183. doi: 10.1016/j.compscitech. 2014.07.015

Shao, Z., and Vollrath, F. (2002). Surprising strength of silkworm silk. Nature 418, 741-741. doi: 10.1038/418741a

Shubhra, Q. T. H., Alam, A. K. M. M., and Beg, M. D. H. (2011). Mechanical and degradation characteristics of natural silk fiber reinforced gelatin composites. Mater. Lett. 65, 333-336. doi: 10.1016/j.matlet.2010.09.059 
Song, R., Ino, H., and Kimura, T. (2009). Mechanical property of silk/bamboo composite paper for effective utilization of waste silk. J. Text. Eng. 55, 85-90. doi: $10.4188 /$ jte. 55.85

Song, R., Kimura, T., and Ino, H. (2010). Papermaking from waste silk and its application as reinforcement of green composite. J. Text. Eng. 56, 71-76. doi: $10.4188 /$ jte. 56.71

Tayeb, A., Amini, E., Ghasemi, S., and Tajvidi, M. (2018). Cellulose nanomaterialsbinding properties and applications: a review. Molecules 23:2684. doi: 10.3390/ molecules23102684

Theng, D., Arbat, G., Delgado-Aguilar, M., Vilaseca, F., Ngo, B., and Mutjé, P. (2015). All-lignocellulosic fiberboard from corn biomass and cellulose nanofibers. Ind. Crops Prod. 76, 166-173. doi: 10.1016/j.indcrop.2015.06.046

Theng, D., El Mansouri, N.-E., Arbat, G., Ngo, B., Delgado-Aguilar, M., Pèlach, M. À, et al. (2017). Fiberboards made from corn stalk thermomechanical pulp and kraft lignin as a green adhesive. Bio Resour. 12, 2379-2393. doi: 10.15376/biores. 12.2.2379-2393

Toprakcioglu, Z., Levin, A., and Knowles, T. P. J. (2017). Hierarchical biomolecular emulsions Using 3-D microfluidics with uniform surface chemistry. Biomacromolecules 18, 3642-3651. doi: 10.1021/acs.biomac.7b01159

Urreaga, J. M., and de la Orden, M. U. (2006). Chemical interactions and yellowing in chitosan-treated cellulose. Eur. Polym. J. 42, 2606-2616. doi: 10.1016/j. eurpolymj.2006.05.002

Vogler, E. A. (1998). Structure and reactivity of water at biomaterial surfaces. $A d v$. Coll. Interface Sci. 74, 69-117. doi: 10.1016/S0001-8686(97)00040-7

Vollrath, F., Porter, D., and Holland, C. (2013). The science of silks. MRS Bull. 38, 73-80. doi: 10.1557/mrs.2012.314

Wang, Y., Cheng, Z., Liu, Z., Kang, H., and Liu, Y. (2018a). Cellulose nanofibers/polyurethane shape memory composites with fast water-responsivity. J. Mater. Chem. B 6, 1668-1677. doi: 10.1039/C7TB03069J

Wang, Y., Wen, J., Peng, B., Hu, B., Chen, X., and Shao, Z. (2018b). Understanding the mechanical properties and structure transition of Antheraea pernyi Silk fiber induced by its contraction. Biomacromolecules 19, 1999-2006. doi: 10.1021/acs. biomac.7b01691

Xiao, L., Liu, S., Yao, D., Ding, Z., Fan, Z., Lu, Q., et al. (2017). Fabrication of silk scaffolds with nanomicroscaled structures and tunable stiffness. Biomacromolecules 18, 2073-2079. doi: 10.1021/acs.biomac.7b00406

Yazawa, K., Malay, A. D., Ifuku, N., Ishii, T., Masunaga, H., Hikima, T., et al. (2018). Combination of amorphous silk fiber spinning and postspinning crystallization for tough regenerated silk fibers. Biomacromolecules 19, 2227-2237. doi: 10. 1021/acs.biomac.8b00232

Yin, Y., Zhao, X., and Xiong, J. (2019). Modeling analysis of silk fibroin/poly( $\varepsilon-$ caprolactone) nanofibrous membrane under uniaxial tension. Nanomaterials 9 , 1149. doi: 10.3390/nano9081149

Yuan, Q., Yao, J., Chen, X., Huang, L., and Shao, Z. (2010). The preparation of high performance silk fiber/fibroin composite. Polymer 51, 4843-4849. doi: 10.1016/j.polymer.2010.08.042

Zhang, Y., Zuo, Y., Wen, S., Hu, Y., and Min, Y. (2018). Distinctive stress-stiffening responses of regenerated silk fibroin protein polymers under nanoscale gap geometries: effect of shear on silk fibroin-based materials. Biomacromolecules 19, 1223-1233. doi: 10.1021/acs.biomac. 8 b00070

Conflict of Interest: The authors declare that the research was conducted in the absence of any commercial or financial relationships that could be construed as a potential conflict of interest.

Copyright (c) 2020 Eom, Park, Jin and Kwak. This is an open-access article distributed under the terms of the Creative Commons Attribution License (CC BY). The use, distribution or reproduction in other forums is permitted, provided the original author(s) and the copyright owner(s) are credited and that the original publication in this journal is cited, in accordance with accepted academic practice. No use, distribution or reproduction is permitted which does not comply with these terms. 